Published in final edited form as:

ACS Nano. 2020 February 25; 14(2): 1360-1368. doi:10.1021/acsnano.9b06875.

\title{
Engineering an Enzyme for Direct Electrical Monitoring of Activity
}

\author{
Bintian Zhang ${ }^{1, \AA}$, Hanqing Deng ${ }^{2, \uparrow, ~ S o h i n i ~ M u k h e r j e e ~}{ }^{2}$, Weisi Song ${ }^{1}$, Xu Wang $^{2}$, Stuart \\ Lindsay ${ }^{1,2,3,{ }^{*}}$ \\ ${ }^{1}$ Biodesign Institute, Arizona State University, Tempe, AZ 85287. \\ ${ }^{2}$ School of Molecular Sciences, Arizona State University, Tempe, AZ 85287. \\ ${ }^{3}$ Department of Physics, Arizona State University, Tempe, AZ 85287.
}

\begin{abstract}
Proteins have been shown to be electrically-conductive if tethered to an electrode by means of a specific binding agent, allowing single molecules to be wired into an electrical sensing circuit. Such circuits allow enzymes to be used as as sensors, detectors and sequencing devices. We have engineered contact points into a $\Phi 29$ polymerase by introducing biotinylatable peptide sequences. The modified enzyme was bound to electrodes functionalized with streptavidin. $\Phi 29$ connected by one biotinylated contact and a second non-specific contact showed rapid small fluctuations in current when activated. Signals were greatly enhanced with two specific contacts. Features in the distributions of DC conductance increased by a factor 2 or more over the open- to closed conformational transition of the polymerase. Polymerase activity is manifested by rapid (millisecond) large (25\% of background) current fluctuations imposed on the DC conductance.
\end{abstract}

\section{Graphical Abstract}

\footnotetext{
*Corresponding Author: stuart.lindsay@ asu.edu.

IlThese authors contributed equally to this work.

Author Contributions

BZ carried out the STM measurements, HD synthesized and characterized the polymerases, SM analyzed data, WS analyzed data and assisted with measurements, XW helped to plan the synthesis of molecules, SL designed experiments, analyzed data and wrote the manuscript.

The authors declare the following competing financial interest(s): SL is a cofounder of a company with interests in this the subject matter of the present paper. SL, BZ and HD are named as co-inventors on patent applications.
} 


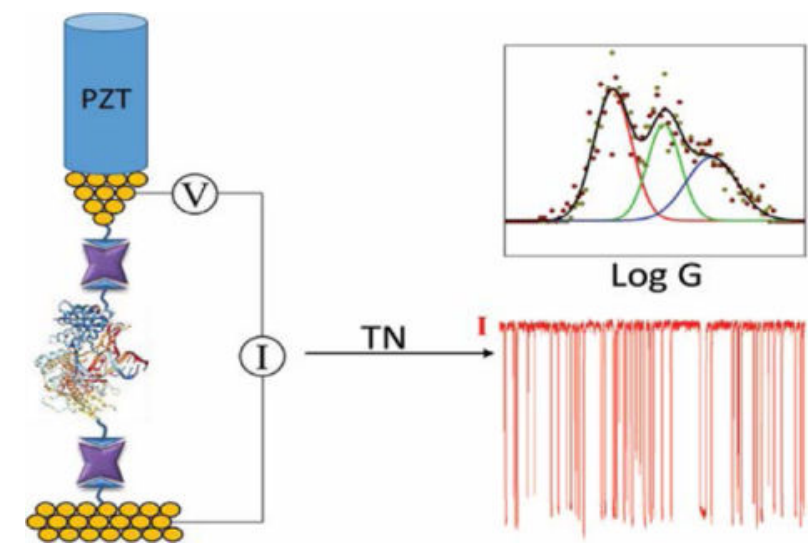

\section{Keywords}

bioelectronics; polymerase activity; single molecule conductance; protein conductivity; bioelectronic circuits

Proteins are widely assumed to be insulators. ${ }^{1,2}$ Reports of metallic conduction in bacterial wires ${ }^{3,4}$ and long range transport in protein multilayers ${ }^{5}$ were thought to be exceptions. Motivated by a theoretical study that suggested that many proteins evolve towards a quantum critical state, ${ }^{6}$ we recently demonstrated that a number of proteins, chosen only for their redox inactivity (i.e. no electron transport function, no redox active centers) conduct very well if contacted by binding agents that can inject charge carriers into their interiors. ${ }^{7,} 8$ This conductance is electron (or hole) mediated because measurements were made under potential control in conditions that eliminate Faradaic currents. ${ }^{8}$ The initial studies used proteins with multiple binding sites (antibodies, streptavidin) to bridge electrodes. However, such attachments limit the degree to which a change on binding a ligand (or an accompanying conformation change) can be studied, because the active sites are tied up as the fixed electrical contact points. In the case of streptavidin, which has four binding sited for biotin, it is possible to use two of the sites as contacts and to study the change of conductance as additional biotin molecules bind. ${ }^{6}$ However, this is a special case, and solving this problem for an arbitrary protein would provide a valuable tool for developing bioelectronic devices in which the chemical versatility of enzymes was exploited by direct electrical measurements. Here, we address the problem of introducing contact points that do not interfere with the active site of a protein, mimicking a mutlivalent protein while retaining the biochemical activity of the original protein. We chose to use $\Phi 29$ polymerase because it is a well-studied ${ }^{9}$ and highly accurate ${ }^{10,11}$ DNA dependent DNA polymerase and already in use as a single-molecule sequencing device. ${ }^{12}$ Here, we demonstrate a technique for inserting binding sites into a $\Phi 29$ polymerase and show that polymerase activity results in rapid conductance fluctuations. 


\section{RESULTS AND DISCUSSION}

\section{Engineering contacts}

Design criteria were: (1) That contact points be remote from the active site of the enzyme so as to not constrain its functional motions; (2) That the sites not change relative spacing over the open to closed conformational transition; (3) That the spacing of the contact points be as large as possible; (4) That the inserted sequences not change the isoelectric point of the enzyme. To implement point (2), that the contact points not move, we looked for atomic sites that had the same crystal-structure coordinates in the two conformations of the polymerase that are associated with its enzymatic activity. ${ }^{13}$ It is important to do this because these points are constrained by the fixed contacts, a constraint that would affect polymerase activity if contact points that moved over the conformational transition were chosen. A large spacing (criterion 3) allows for a larger separation of the contact points, facilitating the construction of junctions. The requirement that the $\mathrm{pI}$ of the engineered protein be close to that of the wild type (criterion 4) is to ensure that protein folding is not altered by an altered distribution of charge on the protein surface. Insertion of sequences that changed the PI significantly rendered the polymerase inactive. Measurements of polymerase activity served to test the viability of the engineered polymerases. In our first iteration, the Avitag peptide sequence was inserted near the N-terminus of the polymerase (Gen I). The lysine in this sequence is biotinylated using the BirA enzyme ${ }^{14,15}$ allowing strong, and specific, binding to Streptavidin. Biotin-bound Streptavidin makes an excellent molecular wire $^{8}$ and also serves to keep the $\Phi 29$ away from the metal electrodes. This is important because $\Phi 29$ has seven surface cysteines, and these can bind directly to a metal electrode, denaturing the polymerase. We made a second version (Gen II) with a second Avitag about 5 $\mathrm{nm}$ distant from the first, at a site in the (inactivated) exonuclease domain. A third version (Gen III) had both contacts as well as a flexible linker next to the N-terminal Avitag. Peptide sequences are given in Figure S1a, and visualizations of Generations I and III are shown in Figures $1 \mathrm{a}$ and $\mathrm{b}$. Formation of the complex with streptavidin was confirmed with a protein gel, and the activity of the complex was demonstrated with a rolling-circle amplification assay (Figures S1b, c). The same assay was used to verify polymerase activity in the buffer used for STM measurements.

\section{Scanning Tunneling Microscope Conductance Measurements}

Measurements were made using an electrochemical scanning tunneling microscope (Pico STM, Agilent) with insulated palladium (Pad) probes $^{16}$ and a Pd substrate, both held under potential control using a salt-bridged reference electrode (Figure. 1c). Electrodes were modified with streptavidin using either a thiolated biotin (SH-biotin) or thiolated streptavidin, and then incubated with a solution of the biotinylated polymerase (Methods). Measurements were made in a reaction buffer containing $\mathrm{MgCl}_{2}$ and tris(2carboxyethyl)phosphine) (TCEP) to prevent polymerase oxidation. Nucleotide triphosphates were added to activate the polymerases. Current-voltage (IV) characteristics were measured using a fixed $\mathrm{Z}$ gap (no servo control) which remained constant to within about $0.1 \mathrm{~nm}$ over 1 minute, as determined by tunnel-current measurements. Drift in the X-Y plane cannot be measured so accurately, but the contact point with a target molecule clearly changes over time. The bias was swept between -0.2 and $+0.2 \mathrm{~V}$ and back again at a rate of $1 \mathrm{~V} / \mathrm{s}$. After 1 
minute, the gap was returned to the set-point value and the cycle was repeated to obtain further IV sweeps. 80\% of these sweeps were linear and reproduced exactly on reversing the sweep direction (Figure 1d). The gradients of these sweeps were used to compile conductance distributions that reflect different types of contacts (Figures 1e,f).

It is important to clarify the fact that, in contrast to many electrochemical conduction processes, the conductance does not depend on scan rate because it is electronic (see Methods). We verified this for the polymerase with a series of repeated scans at different scan speeds. At high speeds (10V/s) there was a significant capacitative current (i.e., hysteresis) but the slopes of the IV curves were unchanged. The capacitative contribution was insignificant at a scan rate of $1 \mathrm{~V} / \mathrm{s}$ which is why this rate was chosen.

The molecular junctions (Figure 1c) were assembled by coating the electrodes with streptavidin, using thiolated streptavidin or wild-type streptavidin in combination with biotinylated electrodes (Methods). Conductance through the streptavidin alone is only observed when the gap is less than $3.5 \mathrm{~nm}$. When biotinylated $\Phi 29$ is added to the liquid cell, high conductance is observed out to a $4.5 \mathrm{~nm}$ gap (Figure S2). We obtained signals out to a $6.5 \mathrm{~nm}$ gap, but with decreased frequency. Since the gap between contact points on the polymerase is $5.5 \mathrm{~nm}$, this observation suggests that a vertical alignment of the streptavidinpolymerase-streptavidin complex is unlikely. Unexpectedly, the monobiotinylated Gen I polymerase gave 2 conductance peaks (Figure 1e). The first peak (at $\sim 0.2 \mathrm{nS}$ ) is characteristic of one specific and one non-specific contact. ${ }^{8}$ The additional peak may reflect interactions between surface cysteines on the polymerase and the electrodes (Figure. S3b). The bis-biotinylated Gen III displays yet another high conductance peak in addition to the two observed in the mon-biotinylated polymerase (Figure 1f). This new high conductance peak has a value $(\sim 5.6 \mathrm{nS})$ that is characteristic of a bridge formed by binding of specific ligands. ${ }^{8}$ The width of the peaks reflects the frequency with which various types of contact are made. This is illustrated by the fact that distributions of conductance obtained from monitoring current at a fixed voltage as the STM gap is left to drift in the XY plane (i.e., at constant height) reproduce the distributions obtained from repeated IV measurements. ${ }^{8}$ The width of the lowest conduction peak is little changed between the case of one contact (Figure 1e) and two contacts (Figure 1f) but it is narrowed considerably in the second peak for the case of two contacts, presumably because binding of the second streptavidin shields some of the surface cysteines from the metal electrode surface and reduces the number of ways that surface cysteines can interact with the metal electrodes (peak widths are listed in Table S1).

It is interesting that connection via biotinylated electrodes gives higher conductance than direct attachment via thiolation of surface residues (Figure. S3a), a phenomenon we have observed previously for conduction through streptavidin alone. ${ }^{8}$ We speculate that the binding of biotin into a deep pocket in the streptavidin provides better injection of carriers into the hydrophobic interior of the protein. ${ }^{7}$

From all of the discussion above, we conclude that the highest conduction peak corresponds to conductance through the polymerase molecules. Accordingly, we next explored the conformational dependence of the electronic conductance. 


\section{Conformational dependence of polymerase conductance}

The active domain of the polymerase resembles a human hand, with a 'thumb' subdomain that holds the DNA, a 'palm' subdomain that contains the catalytic site, and a moving 'fingers' subdomain that closes around the complex of DNA template once the correct complementary nucleotide triphosphate (dNTP) is bound. The enzyme is normally 'open' 13 and remains so after binding DNA containing a primer strand and template strand with a 5' overhang. Once the correct dNTP is bound, the fingers close to complete the reaction, opening again only for long enough to bind the next complementary dNTP. ${ }^{13}$ This transient opening can be suppressed by using non-hydrolyzable dNTPs (NH-dNTPs) in which a carbon replaces an oxygen in the triphosphate. ${ }^{17}$

We repeated measurements of the conductance distributions: (a) With a saturating $(1 \mu \mathrm{M})$ concentration ${ }^{18}$ of a single-stranded template with a 15 basepair hairpin primer (Figure S4c). (b) With the template-bound polymerase in the presence of a saturating concentration ${ }^{18}$ $(1 \mathrm{mM})$ of dNTPs. (c) In the presence of a saturating concentration ${ }^{18}(1 \mathrm{mM})$ of NH-dNTPs. The dissociation constant for $\Phi 29$-template interactions is $80 \mathrm{nM} .{ }^{19}$ The Michaelis-Menten constant for dNTP binding to template-bound polymerase is between 5 and $30 \mu \mathrm{M} .{ }^{18,19} \mathrm{All}$ measurements were made in the presence of $10 \mathrm{mM} \mathrm{MgCl}_{2}$. The corresponding conductance distributions are shown in Figures $2 \mathrm{a}, \mathrm{b}$ and $\mathrm{c}$. The distribution in the presence of bound template (Figure 2a) is almost identical to the distribution in the absence of template (Figure 1f - uncertainties in these fits are discussed in the caption and listed in Table S1). On addition of dNTPs there are large shifts in the conductance peaks (Figure 2b-1.3x, 2.3x and $2 \mathrm{x}$ for peaks 1,2 and 3 ). Locking the polymerase in the closed form changes the peak positions a little more (Figure 1c, 1.8x, 3.2x and 2.3x) with a notable sharpening of the third peak (see Figure S5 for replication of this sharp peak, and Table S1 for a list of fitted peak widths). We speculate that the "unlocked" but dNTP bound polymerase (Figure $2 b$ ) may be fluctuating rapidly between sub conformations, broadening the high conductance peak, which then narrows substantially as the closed conformation is locked in with non hydolyzable dNTPs (Figure 2c). We conclude that the open to closed transition of the polymerase is accompanied by large changes in conductance.

These measurements were taken in the presence of $\mathrm{Mg}^{2+}$ so the polymerase is catalytically active in the presence of both template DNA and dNTPs. This is marked by additional noise, as shown in samples of the IV curves for the three cases in Figures 2d,e and f. The inactive polymerase (Figures. 2d,f) does not display large noise spikes in the bias region below \pm $100 \mathrm{mV}$, as reported for other proteins. ${ }^{8}$ (Above $100 \mathrm{mV}$, the electric field at the contact points induces telegraph noise - the current at a fixed bias switches between two distinct levels. ${ }^{8}, 20$ ) However, when the polymerase is active (Figure 2e) large spikes are also observed in the bias region below $\pm 100 \mathrm{mV}$.

\section{Noise Measurements}

These observations indicate that rapid polymerase activity can be followed by recording current versus time $(\mathrm{I}(\mathrm{t}))$ at a bias below $100 \mathrm{mV}$ in the presence of template-bound $\Phi 29$, $\mathrm{Mg}^{2+}$ and dNTPs. We set a gap of $2.5 \mathrm{~nm}$ under servo control, opened the servo, increased the gap to $6 \mathrm{~nm}$, then brought the tip down to $4.5 \mathrm{~nm}$ and recorded current for $60 \mathrm{~s}$ at a bias 
of $50 \mathrm{mV}$. Typically, no current was recorded for the first 10-20 s, after which a contact formed and an I(t) curve was obtained. Contacts were formed with molecules in $>50 \%$ of these "fishing" attempts. The currents jumped suddenly on contact with the molecule, but then changed substantially as the contact point drifted. A typical current-time trace is shown in Figure 3a. This variation in current vs. time was shown to be a consequence of the drift in the contact point - the distribution of currents taken in an $\mathrm{I}(\mathrm{t})$ curve replicates the distribution measured by taking IV curves from many different contact points. ${ }^{8}$

Telegraph noise $(\mathrm{TN})$ is clearly visible in $\mathrm{I}(\mathrm{t})$ traces taken with activated polymerases. Figure S6 shows examples for the Gen I, single contact polymerase. (The TN is aperiodic and therefore does not contribute well-defined features to a power spectrum of the signal.) However, these data also illustrate how difficult it is to quantify the noise in the presence of a rapidly varying background current.

We removed the variable background using an asymmetric least squares (ALS) fit. ${ }^{21}$ The ALS accurately follows the background without distorting the noise signals (Figure S7). The $\mathrm{I}(\mathrm{t})$ trace shown in Figure $3 \mathrm{a}$ was obtained with a $\mathrm{dA}_{10}$ template (Figure $\mathrm{S} 4 \mathrm{~b}$ ) in the presence of dTTP. The raw data are shown as black points, largely obscured by the ALS fit (red). The subtracted signal, corresponding to the fluctuations, is shown in Figure $3 \mathrm{~b}$. When the same procedure is applied to a trace taken at about the same current in the absence of dTTP (Figures $3 \mathrm{c}$ and d) it is clear that noise is also present in the -dTTP control. However the noise is much smaller in amplitude. A closer examination of the signals reveals two distinct levels of telegraph noise, as shown in Figure 4a and labeled "SF" (small fluctuations) and "LF" (large fluctuations). Noise-amplitude distributions for the traces in Figures $3 b$ and d are shown in Figure 4b. The SF appear in all measurements whereas the LF are only present when the polymerase is active. Quantifying this qualitative observation is complicated because the absolute amplitude of the fluctuations depends on the background current, and this current varies over a run. However, the run-to-run variations are generally much larger, so we obtained an approximate measure of the relative fluctuation size as follows: For each molecule measured, we binned the ALS fitted baseline currents as shown by the examples in Figures S8a,c. Many of these distributions could be fitted by a Gaussian (red curve). Many could not - for example the background can jump between two or three levels. In these cases we fitted the largest peak that was clear of the background. The peak of the fitted Gaussian, $\mathrm{I}_{\mathrm{p}}$, was then used to characterize the baseline for that run. Example of the binned noise signals are given in in Figures S8b,d. To characterize these binned distributions we used a double exponential distribution in which one parameter $\left(i_{S}\right)$ characterizes the amplitude distribution of the small fluctuations and another parameter $\left(i_{L}\right)$ characterizes the amplitude distribution of the large fluctuations:

$$
N(i)=A_{1} \exp \left(-\frac{i}{i_{S}}\right)+A_{2} \exp \left(-\frac{i}{i_{L}}\right)
$$

where $i$ is the current in a given bin of the current distribution. In the case of the experiments in which dTTP was withheld (e.g., Figure S8b), the fits all converged to a single exponential $\left(\left(i_{S}=i_{L}\right)\right.$. For recordings with dTTP present, most of the fits converged on the double exponential distribution with $i_{S}<i_{L}$ (4 of 13 molecules showed the small fluctuations only in 
the presence of dTTP). The results are summarized in Figure 4c. Most activated molecules (+dTTP) showed both large (red points) and small (blue points) fluctuations. The controls (-dTTP) showed only small fluctuations (green points), essentially equal to the small fluctuations also seen in the activated polymerases. There is an approximately linear relation between background current and fluctuation amplitude as shown by the three linear fits. For the large fluctuations, characteristic of active polymerases, a typical (1/e value) of current is $i_{L}=(0.25 \pm 0.026) i_{p}$ For the small fluctuations, present in both active an inactive polymerases, a typical value is $i_{L}=(0.06 \pm 0.01) i_{p}$. Thus the active state can be identified by the presence of fluctuations that are about $25 \%$ of the baseline current, while fluctuations in the inactive state are about $6 \%$ of the baseline current. Not all polymerase molecules contacted were active as indicated by the lack of large fluctuations in 4 of the 13 molecules studied (these four data points are evident as the extra four blue data points for the SF in the presence of dTTP in Figure 4C that do not have corresponding red data points for the LF). On the other hand, none of the eight -dTTP control runs showed large fluctuations (Table 1).

This analysis was repeated using data obtained in 38 runs with a d(ATC) 5 template (Figure S4d) and 25 runs with a d(C) 10 template (Figure S4a). The results are summarized in Figures S9a and $b$. The fitted amplitude distributions for the large fluctuations (LF) show considerable variation, but the trends observed for $\mathrm{d}(\mathrm{A})_{10}$ (Figure $4 \mathrm{c}$ ) are reproduced well with $i_{L}=0.27( \pm 0.03) \mathrm{I}_{\mathrm{p}}$ and $i_{S}=0.04( \pm 0.01) \mathrm{I}_{\mathrm{p}}$ for d(ATC $)_{5}$ and $i_{L}=0.32( \pm 0.03) \mathrm{I}_{\mathrm{p}}$ and $i_{S}=$ $0.05( \pm 0.007) \mathrm{I}_{\mathrm{p}}$ for $\mathrm{d}(\mathrm{C})_{10}$. In order to confirm the association of large fluctuations (amplitudes $>25 \%$ of the baseline current) with polymerase activity we carried out experiments with several DNA templates (Figure S4) in different conditions. Active ("A" in Table 1) polymerases were measured in the reaction buffer ( $1 \mathrm{mM}$ phosphate buffer, $\mathrm{pH}=7.4$, $4 \mathrm{mM}$ TCEP, $10 \mathrm{mM} \mathrm{MgCl} 2$ with $1 \mathrm{mM}$ dNTPs and $1 \mu \mathrm{M}$ template). In the control experiments ("C" in Table 1), one essential ingredient was withheld. In addition, we carried out measurements using nonhydrolyzable dNTPs. Fluctuations were analyzed as described above.

Clearly, withholding any one of the ingredients critical to polymerase function abolishes the large fluctuations. An interesting exception was $(\mathrm{dA})_{10}(\mathrm{dC})_{10}$ in the presence of dTTP only. We expected that the polymerase would reach the end of the A tract and then stall for want of the missing dGTP nucleotide, but about a third of the molecules appear to be active for at least some part of the recording. We noted that the telegraph noise obtained from the (ATC) and $\mathrm{C}_{10}$ templates was generally more regular in time and amplitude than was the case for the $\mathrm{A}_{10}$ template (Figures $5 \mathrm{a}, \mathrm{b}, \mathrm{c}$ ) and a denaturing gel of the products of polymerization (Figure $5 \mathrm{~d}$ ) clearly shows incomplete transcription of the $\mathrm{A}_{10}$ template (red arrow, lane 7). Thus it seems likely that the polymerase falls off the A homopolymer tract, allowing another template to bind, and accounting for the activity seen for the $\mathrm{A}_{10} \mathrm{C}_{10}$ template in the absence of dGTP.

\section{CONCLUSIONS}

These findings show that enzyme activity can be monitored via a direct electrical measurement, opening the way for integrating the analytical power of enzymes into integrated circuits, once a reliable way is found to integrate the molecules into solid-state 
gap devices. ${ }^{20}$ Engineering two contact points into a polymerase yields features in the conductance distribution that are approximately 3 to 10 times larger than those observed with only one engineered contact and a second, non-specific, contact. The conductance of the complex of streptavidin and doubly biotinylated $\Phi 29$ is further increased if biotin is used to anchor the streptavidin to the electrodes, instead of thiolated surface lysines. There are large changes in the conductivity distribution as the polymerase undergoes the open to closed transition. In addition, polymerase activity is marked by rapid noise spikes that have an amplitude of about $25 \%$ (or more) of the background current, distinct from the smaller (6\% of background) signals present in both active and inactive polymerase. Further understanding of these signals will require investigation of molecules wired into solid-state gap devices, for which much longer and less variable data runs will be obtained. We have made devices in which a fixed junction is created by drilling an orifice through a stack of metal-dielectric-metal layers ${ }^{22}$ and we are exploring the adaptation of these devices as fixed contacts for enzyme measurements. With such devices we hope to be able to get data that is free from interruptions owing to contact drift, so that we can determine if the pauses observed in the signals (Figure $3 \mathrm{~b}$ ) are intrinsic to the polymerase or not. Furthermore, a steady contact would allow much longer trains of data to be gathered, so that pauses caused by slower binding of dNTPs and template could be used to explore polymerase function in experiments in which the concentration of each component was reduced systematically.

\section{METHODS/EXPERIMENTAL}

\section{Recombinant Ф29 DNA polymerase constructs with inserted Avitag}

The starting enzyme was a $\Phi 29$ DNA polymerase, rendered exonuclease-deficient with D12A and D66A mutations. A Q5 site-directed mutagenesis kit (NEB) was used to insert the Avitag DNA sequence into a pET15b plasmid containing the mutant polymerase gene. The equivalent inserted peptide sequence is shown in blue, with flanking linker sequences in yellow, in Figure S1a. The epsilon-amine of the central lysine (K, marked by red arrow on Fig. S1a) was biotinylated using the BirA enzyme. ${ }^{14}$ Three generations of modified enzyme were tested. The first (Gen 1) was biotinylated only at the $\mathrm{N}$ terminus of the protein. The second (Gen II) contained a second Avitag 5nm from the N terminus between E279 and D280. This second site is located in the deactivated exonuclease domain and was chosen because its position does not change with respect to the $\mathrm{N}$ terminus over the open to closed transition. ${ }^{23,} 24$ The third (Gen III) contained an additional flexible linking sequence (GNSTNGTSNGSS) adjacent to the N-terminal Avitag to allow for greater flexibility in contact geometry. Biotinylation was verified with a SDS-PAGE gel analysis of the free- and streptavidin bound polymerases. Figure S1b shows the increase in molecular weight that occurs as streptavidin binds to the Gen III polymerase (yet higher molecular weight features are likely polymer aggregates of alternating polymerase and streptavidin). The activity of the modified polymerases was verified in vitro using a rolling circle amplification assay (Figure S1c). Further details are given in the caption for Figure S1a.

\section{Functionalizing substrates and STM probes}

Palladium substrates were prepared by evaporating a $200 \mathrm{~nm}$ palladium film onto a silicon wafer using an electron-beam evaporator (Lesker PVD 75), with a $10 \mathrm{~nm}$ titanium adhesion 
layer. The substrates were treated with a hydrogen flame immediately before functionalizing and then immersed in solutions of thiolated streptavidin (ProteinMods) or thiolated biotin overnight. The thiolated biotin was prepared as described elsewhere ${ }^{8}$ and dissolved in freshly degassed pure ethanol to a final concentration of $50 \mu \mathrm{M} .1 \mu \mathrm{M}$ thiolated streptavidin solutions in $1 \mathrm{mM}$ PB buffer were used for substrate functionalization. All the buffers and solutions were prepared in Milli-Q water with a conductivity of 18.2 M $\Omega$. For all measurements, the $1 \mathrm{mM}$ PB buffer ( $\mathrm{pH}$ 7.4) was degassed with argon to avoid interference from oxygen. The polymerization buffer was $1 \mathrm{mM}$ phosphate buffer, $\mathrm{pH}=7.4,4 \mathrm{mM}$ TCEP, $10 \mathrm{mM} \mathrm{MgCl}_{2}$ with $1 \mathrm{mM}$ dNTPs and $1 \mu \mathrm{M}$ template (activity in this buffer was verified with a rolling circle amplification assay). Substrate functionalization was characterized by Fourier transform infrared (FTIR) spectroscopy (Figure. S10). STM probes were etched from a $0.25 \mathrm{~mm}$ Pd wire (California Fine Wires) by an AC electrochemical method. To avoid current leakage, probes were insulated with high-density polyethylene following the method described earlier for gold probes. ${ }^{16}$ Each probe was tested by STM in $1 \mathrm{mM}$ phosphate buffer ( $\mathrm{pH} 7.4$ ) at $+0.5 \mathrm{~V}$ bias to ensure the leakage current was $<1 \mathrm{pA}$. For functionalization, the probe was immersed in ligand solutions for $4 \mathrm{~h}$ or overnight, then rinsed with water, dried with nitrogen gas, and used immediately. Cyclic voltammetry was used to check that the potential regions used ( +50 to $-50 \mathrm{mV}$ vs $\mathrm{Ag} / \mathrm{AgCl}, 10 \mathrm{mM} \mathrm{KCl}$ ) were free from Faradaic currents in the presence of the various components of the assembly (Figures S11$13)$.

\section{STM Measurements}

STM measurements were carried out on a PicoSPM scanning probe microscope (Agilent Technologies), using a DAQ card (PCI-6821 or PCIE-7842R, National Instruments) for data acquisition. The Teflon liquid cell was cleaned with Piranha solution and sonicated in MilliQ water (Note that Piranha solution is highly corrosive and must be handled with extreme care). $\mathrm{An} \mathrm{Ag} / \mathrm{AgCl}$ reference electrode with a $10 \mathrm{mM} \mathrm{KCl}$ salt bridge was connected onto the substrate. This keeps both electrodes in the double-layer region of potential where no Faradaic currents flow. ${ }^{8}$ Protein conformations can be affected by the double-layer electric field ${ }^{25}$ but measurements showed little effect from small changes in surface potential, typical of the potentials used here. ${ }^{8}$ The probe was initially engaged at a $4 \mathrm{pA}$ setpoint current with a bias of $-0.2 \mathrm{~V}$ and left to stabilize for $2 \mathrm{~h}$ before measurement. IV sweep and $\mathrm{I}(\mathrm{t})$ measurements are described in detail in reference ${ }^{8}$. The scan-rate dependence of protein conductance was previously studied in detail using an antibody (antidinitrophenol) ${ }^{8}$ but these experiments were not reported in the original paper. For that reason we show the results in Figure S14.

\section{Supplementary Material}

Refer to Web version on PubMed Central for supplementary material.

\section{ACKNOWLEDGMENTS}

This work was supported by grants HG006323 and HG010522 from the National Human Genome Research Institute, by Recognition AnalytiX Corp and the Edward and Nadine Carson Endowment. We thank P. Zhang and W. Konigsberg for useful discussions. 


\section{REFERENCES}

1. Nitzan A, Chemical Dynamics in Condensed Phases. Oxford University Press: Oxford, 2006.

2. Bostick CD; Mukhopadhyay S; Pecht I; Sheves M; Cahen D; Lederman D, Protein Bioelectronics: a Review of What We Do and Do Not Know. Rep. Prog. Phys 2018, 81, 026601-026658. [PubMed: 29303117]

3. Adhikari RY; Malvankar NS; Tuominen MT; Lovley DR, Conductivity of Individual Geobacter Pili. RSC Adv 2016, 6, 8354-8357.

4. Meysman FJR; Cornelissen R; Trashin S; Bonne R; Martinez SH; van der Veen J; Blom CJ; Karman C; Hou JL; Eachambadi RT; Geelhoed JS; Wael K; Beaumont HJE; Cleuren B; Valcke R; van der Zant HSJ; Boschker HTS; Manca JV, A Highly Conductive Fibre Network Enables CentimetreScale Electron Transport in Multicellular Cable Bacteria. Nat. Commun 2019, 10, 4120-4128.

5. Nadav Amdursky; Debora Marchak; Lior Sepunaru; Israel Pecht; Mordechai Sheves; Cahen D, Electronic Transport via Proteins. Adv. Mater 2014, 26, 7142-7161. [PubMed: 25256438]

6. Vattay G; Salahub D; Csabai I. a.; Nassimi A; Kaufmann SA, Quantum Criticality at the Origin of Life. J. Phys. : Conference Series 2015, 626, 012023012033.

7. Zhang B; Lindsay S, Electronic Decay Length in a Protein Molecule. Nano letters 2019, 19, 40174022. [PubMed: 31144824]

8. Zhang B; Song W; Pang P; Lai H; Chen Q; Zhang P; Lindsay S, The Role of Contacts in LongRange Protein Conductance. Proc. Natl. Acad. Sci. U S A 2019, 116, 5886-5891. [PubMed: 30846548]

9. Blanco L; Bernad A; Lazaro JM; Martin G; Garmendia C; Salas M, Highly Efficient DNA Synthesis by the Phage Phi 29 DNA Polymerase. Symmetrical Mode of DNA Replication. J. Biol. Chem 1989, 264, 8935-8940. [PubMed: 2498321]

10. Blanco L; Salas M, Relating Structure to Function in Phi29 DNA Polymerase. J. Biol. Chem 1996, 271, 8509-8512. [PubMed: 8621470]

11. Esteban JA; Salas M; Blanco L, Fidelity of Phi 29 DNA Polymerase. Comparison Between Protein-Primed Initiation and DNA Polymerization. J. Biol. Chem 1993, 268, 2719-2726. [PubMed: 8428945]

12. Korlach J; Bibillo A; Wegener J; Peluso P; Pham TT; Park I; Clark S; Otto GA; Turner SW, Long, Processive Enzymatic DNA Synthesis Using 100\% Dye-Labeled Terminal Phosphate-Linked Nucleotides. Nucleosides, Nucleotides Nucleic Acids 2008, 27, 1072-1083. [PubMed: 18711669]

13. Steitz TA, A Mechanism for All Polymerases. Nature 1998, 391 (6664), 231-232. [PubMed: 9440683]

14. Fairhead M; Howarth M, Site-Specific Biotinylation of Purified Proteins Using BirA. Methods Mol. Biol 2015, 1266, 171-84. [PubMed: 25560075]

15. Beckett D; Kovaleva E; Schatz PJ, A Minimal Peptide Substrate in Biotin Holoenzyme SynthetaseCatalyzed Biotinylation. Protein Sci 1999, 8, 921-929. [PubMed: 10211839]

16. Tuchband M; He J; Huang S; Lindsay S, Insulated Gold Scanning Tunneling Microscopy Probes for Recognition Tunneling in an Aqueous Environment. Rev. Sci. Instrum 2012, 83, 015102 015106.

17. Beard WA; Shock DD; Batra VK; Pedersen LC; Wilson SH, DNA Polymerase Beta Substrate Specificity: Side Chain Modulation of the "A-Rule". J. Biol. Chem 2009, 284, 31680-31689. [PubMed: 19759017]

18. Morin JA; Cao FJ; Lazaro JM; Arias-Gonzalez JR; Valpuesta JM; Carrascosa JL; Salas M; Ibarra B, Mechano-Chemical Kinetics of DNA Replication: Identification of the Translocation Step of a Replicative DNA Polymerase. Nucleic Acids Res 2015, 43, 3643-3652. [PubMed: 25800740]

19. Lazaro JM; Blanco L; Salas M, Purification of Bacteriophage Phi 29 DNA Polymerase. Methods Enzymol 1995, 262, 42-49. [PubMed: 8594366]

20. Zhang B; Song W; Pang P; Zhao Y; Zhang P; Csabai I; Vattay G; Lindsay S, Observation of Giant Conductance Fluctuations in a Protein. Nano Futures 2017, $1035002-0350017$.

21. Peng J; Peng S; Jiang A; Wei J; Li C; JieTana, Asymmetric Least Squares for Multiple Spectra Baseline Correction. Anal. Chim. Acta 2010, 683, 63-68. [PubMed: 21094382] 
22. Pang P; Ashcroft BA; Song W; Zhang P; Biswas S; Qing Q; Yang J; Nemanich RJ; Bai J; Smith JT; Reuter K; Balagurusamy VSK; Astier Y; Stolovitzky G; Lindsay S, Fixed-Gap Tunnel Junction for Reading DNA Nucleotides. ACS Nano 2014, 8, 11994-12003. [PubMed: 25380505]

23. Berman AJ; Kamtekar S; Goodman JL; Lazaro JM; de Vega M; Blanco L; Salas M; Steitz TA, Structures of Phi29 DNA Polymerase Complexed with Substrate: the Mechanism of Translocation in B-family Polymerases. EMBO J 2007, 26, 3494-505. [PubMed: 17611604]

24. Kamtekar S; Berman AJ; Wang J; Lazaro JM; de Vega M; Blanco L; Salas M; Steitz TA, Insights into Strand Displacement and Processivity from the Crystal Structure of the Protein-Primed DNA Polymerase of Bacteriophage Phi29. Mol. Cell 2004, 16, 609-618. [PubMed: 15546620]

25. Ghisellini P; Caiazzo M; Alessandrini A; Eggenhoffner R; Vassalli M; Facci P, Direct Electrical Control of IgG Conformation and Functional Activity at Surfaces. Sci. Rep 2016, 6, 37779-37797. [PubMed: 27883075] 
a

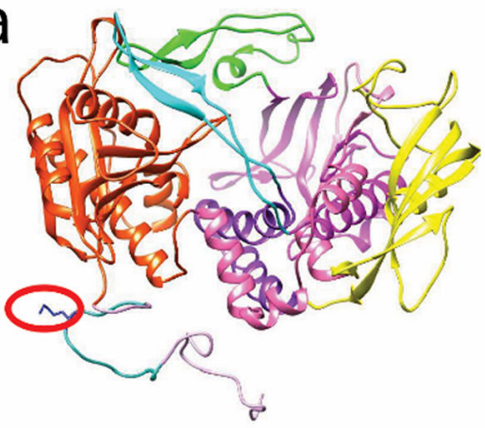

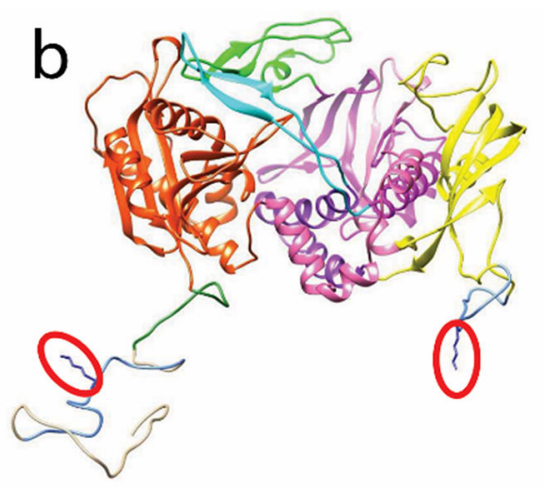
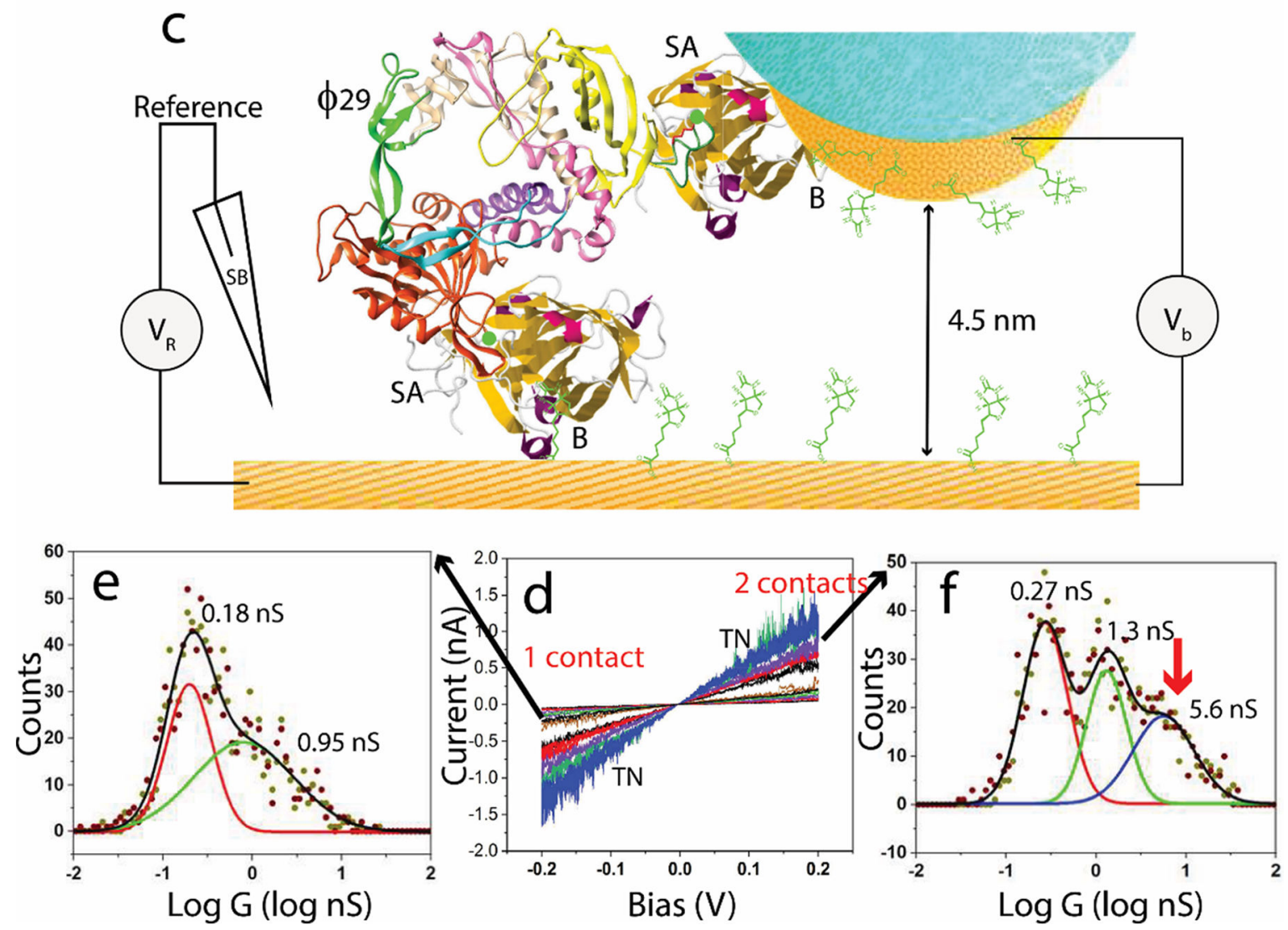

Figure 1: Conductance of polymerases with one and two biotinylated contact points.

(a) 29 polymerase with a single Avitag at the $\mathrm{N}$ terminus (Gen I). Biotinylatable lysine is labeled by the red outline. (b) $\Phi 29$ polymerase with a second Avitag inserted between E279 and D280 and a flexible loop at N terminus (Gen III). (c) An STM probe is held $\sim 4.5 \mathrm{~nm}$ above a conducting substrate, immersed in electrolyte and under potential control via a salt bridge ("SB") to an $\mathrm{Ag} / \mathrm{AgCl}$ reference. Electrodes functionalized with thiolated biotin ("B") capture streptavidin molecules ("SA") which trap a biotinylated polymerase ("Ф29"). (d) Typical current-voltage curves (trace and retrace are superimposed). Conductances for individual molecules are obtained from the slopes of these traces. "TN" indicates region of contact-field induced fluctuations. A doubly biotinylated polymerase has a new high conductance feature at $\sim 6 \mathrm{nS}$ in the conductance distribution (red arrow in $\mathrm{f}$ ) not present in 
the singly-biotinylated molecule (e). The largest uncertainty in the fitted peaks is \pm 0.05 in $\log \mathrm{G}$, corresponding to about $\pm 0.12 \mathrm{G}$ in $\mathrm{G}$. Peak widths and fitting errors for each peak shown here are listed in Table $\mathrm{S} 1$. 

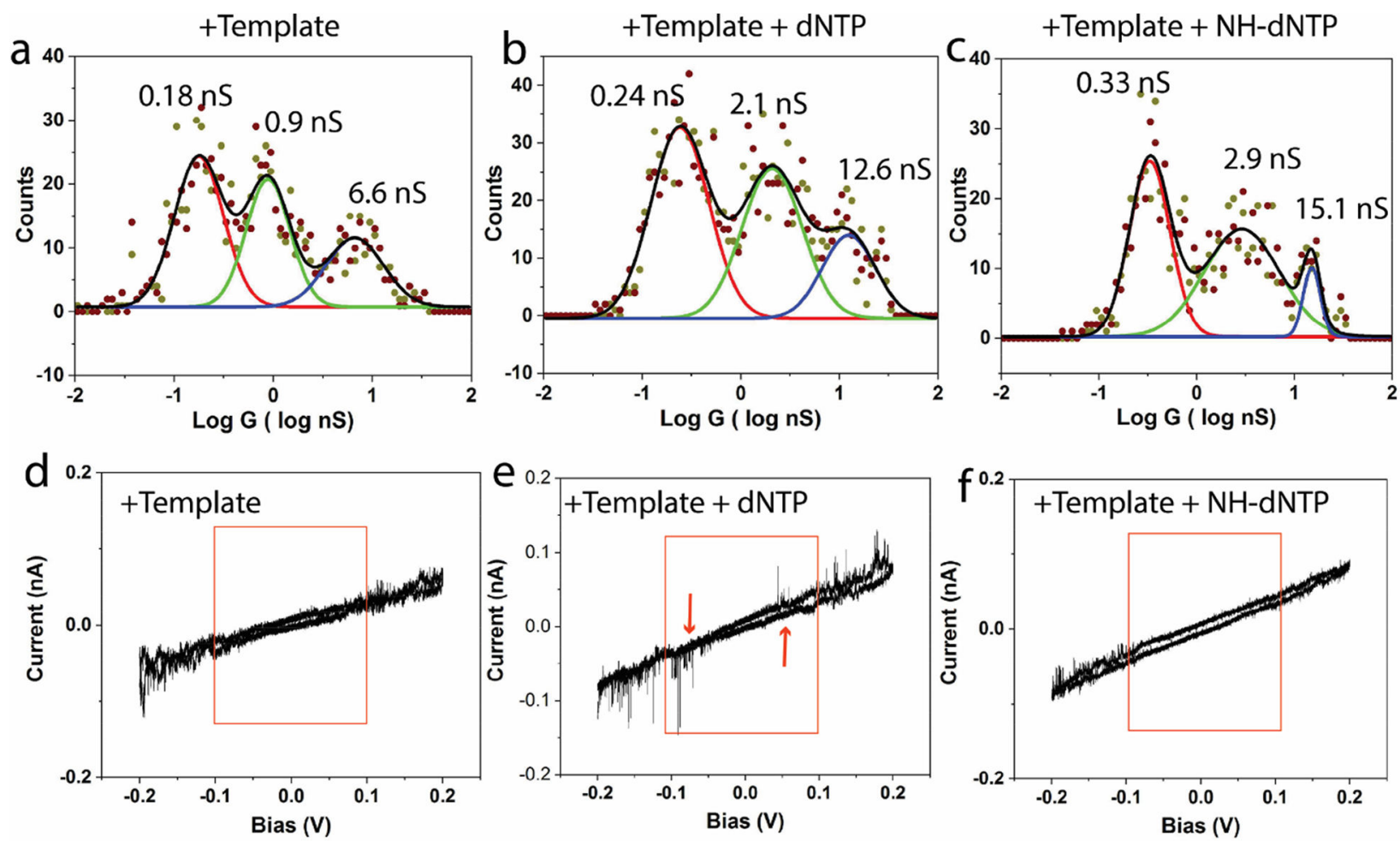

Figure 2: The open to closed transition changes polymerase conductance.

(a) Distribution in the absence of dNTPs but with bound template. The polymerase is largely open. (b) With dNTPs added (mostly closed) the distribution changes dramatically. (c) Conductance distribution for a polymerase locked in the closed conformation with nonhydrolyzable dNTPs. Peak widths and fitting errors for each peak shown here are listed in Table S1. (d,e,f) In the inactive state (-dNTP d, or + NH-dNTP f) the IV curves are noise free in the bias range below $\pm 100 \mathrm{mV}$ (red box). The active polymerase (e) shows noise spikes on the IV curve in this otherwise quiet region (red arrows). All measurements were made with $10 \mathrm{mM} \mathrm{MgCl}_{2}$. 

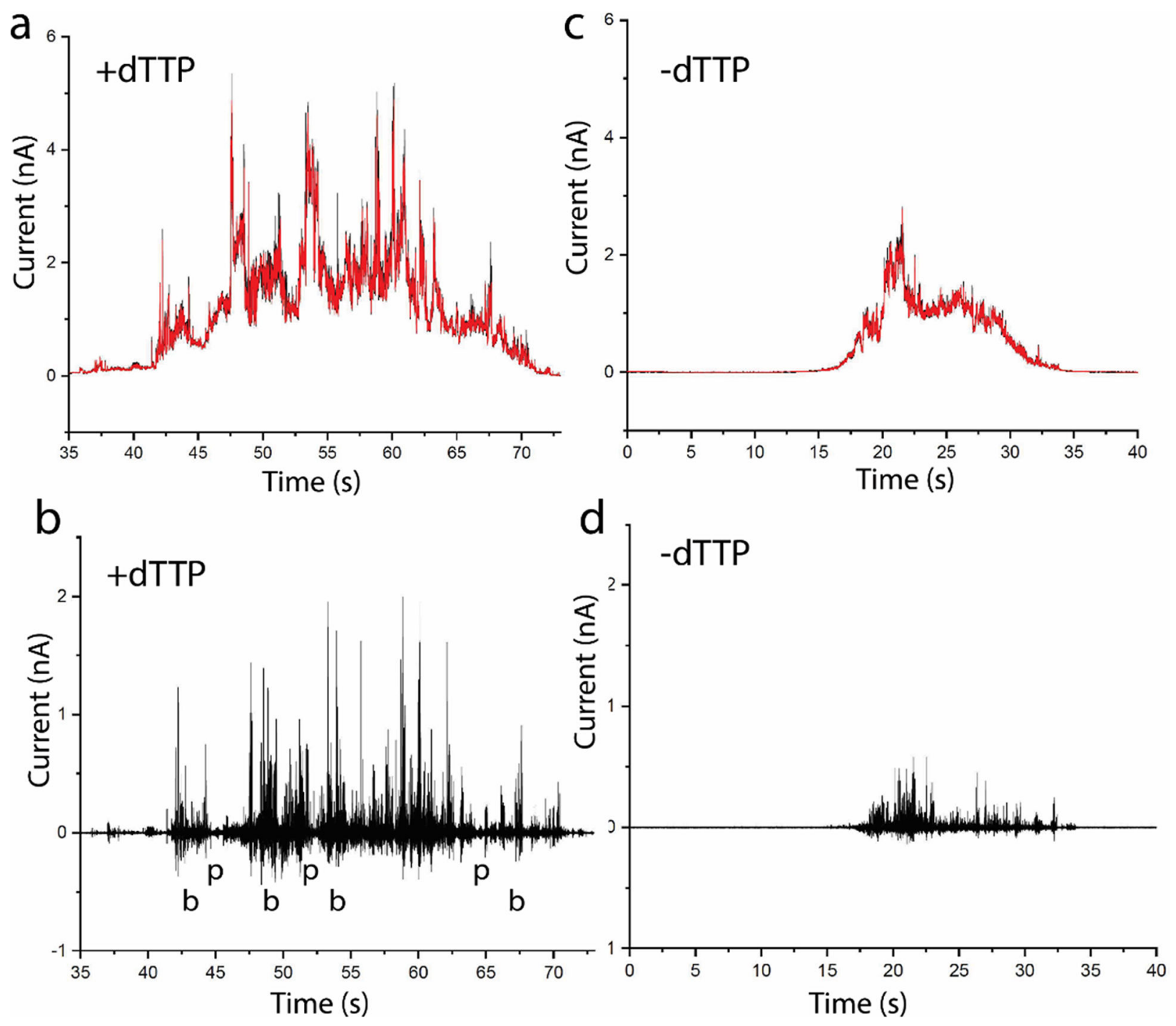

Figure 3: Separating signal from background conductance changes.

(a) The current through the polymerase changes markedly over time as the STM probe drifts (mainly obscured black curve). This drifting baseline current is fitted with an asymmetric least squares (ALS) procedure to yield a smoothed background current (red curve superimposed on black curve). (b) Subtraction of the fitted background shows the rapid changes in current that occur in an activated polymerase. Typically the dynamic signals occur in bursts (b) interspersed with pauses (p). (c) Shows the current recorded vs time for this $\mathrm{dA}_{10}$ template in the absence of dTTP. The ALS fit is shown in red. (d) The baseline subtracted signal shows that there is also noise in the inactive system, though smaller in amplitude than that for the activated polymerase (these data sets $(\mathrm{a}, \mathrm{c})$ were chosen to have about the same DC conductance). 

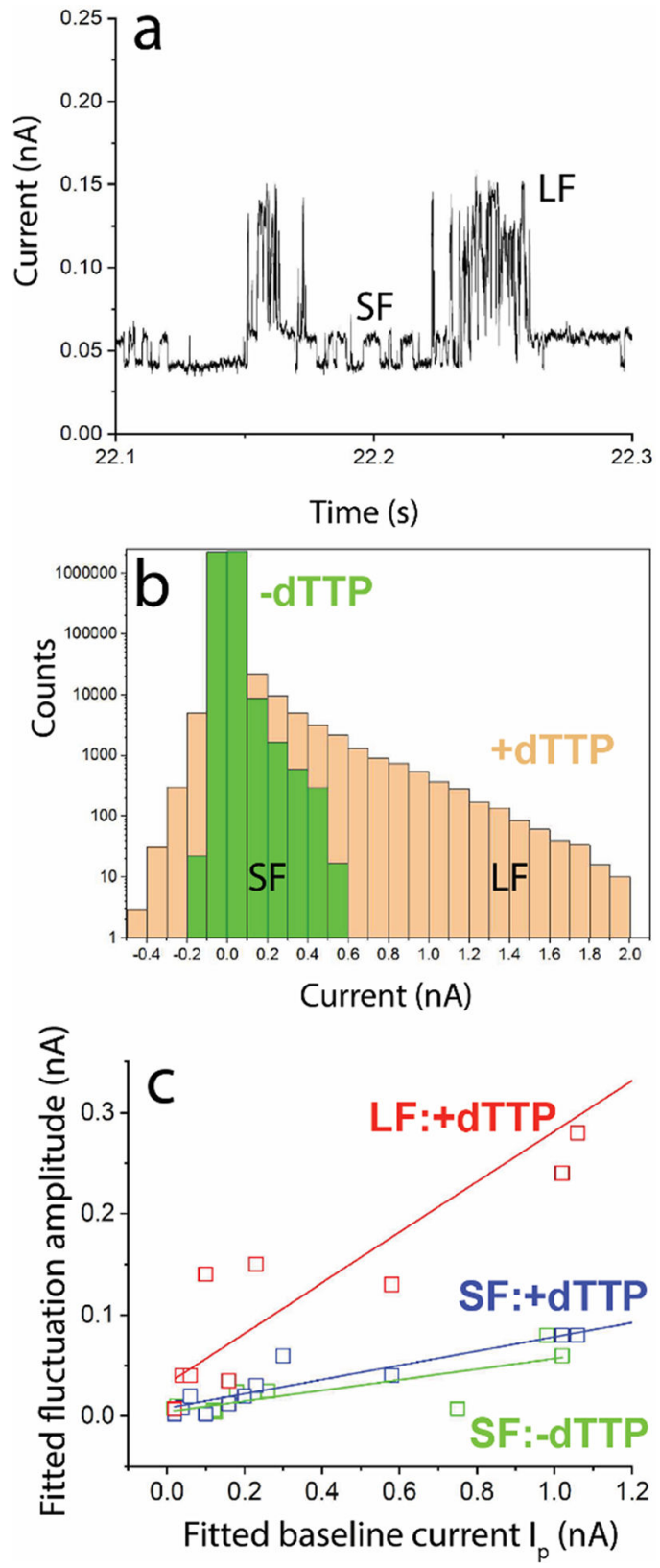

Figure 4: Characterizing the signals generated when polymerase is activated.

(a) An expanded portion of the noise signal measured from an activated polymerase $\left(\mathrm{A}_{10}\right.$ template) showing two distinct noise components - large fluctuations (LF) and small fluctuations (SF). (b) Distributions of noise signal amplitudes from the active (+dTTP) and inactive (-dTTP) polymerase in runs of about the same background current. (c) The peak noise amplitudes, $\mathrm{I}_{\mathrm{L}}(\mathrm{LF})$ and $\mathrm{I}_{\mathrm{S}}(\mathrm{SF})$, depend on the baseline current $\mathrm{I}_{\mathrm{P}}$. $\mathrm{I}_{\mathrm{L}}$ (red) and $\mathrm{I}_{\mathrm{S}}$ (blue) are plotted vs the associated value of baseline current $\left(\mathrm{I}_{\mathrm{P}}\right)$. Only SF are observed in inactive (-dTTP) polymerases (Green). Similar results for other template sequences are shown in Figure S10. In the presence of dTTP, 13 samples showed SF (blue points) but only 9 showed LF as well, indicating that 4 molecules were inactive. 

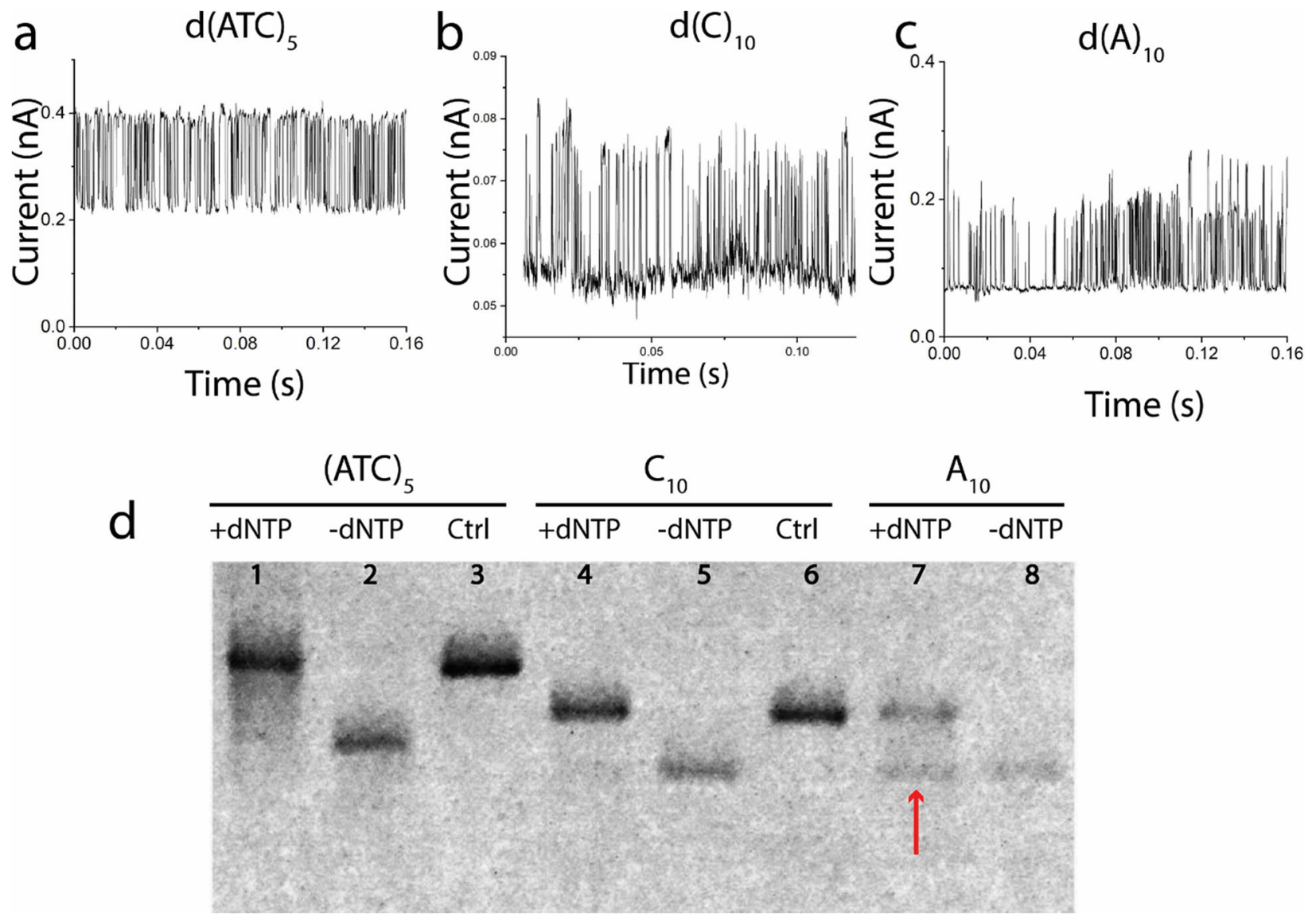

Figure 5: Telegraph noise signals reflect the ease with which a template is processed.

Typical telegraph noise signals (selected from regions of constant baseline current) for $\mathrm{d}(\mathrm{ATC})_{5}$ and $\mathrm{dC}_{10}$ are fairly uniform in amplitude and in the period between signal features. Signals for $\mathrm{dA}_{10}$ are typically more irregular (c). A denaturing gel (d) shows that the $\mathrm{d}(\mathrm{ATC})_{5}$ and $\mathrm{dC}_{10}$ templates are completely converted to full length molecules (the Ctrl lanes are for synthesized fully extended molecules). This is not the case for the $\mathrm{dA}_{10}$ (red arrow) for which polymerization is only partially successful (The $\mathrm{C}_{10}$ control serves as a length control for this molecule also). 
Table 1:

Occurrence of large fluctuations (fraction of measured molecules) for various experimental conditions. $\mathrm{NH}=$ non-hydrolyzable dNTPs. Large fluctuations are identified by a fit converging on a two component distribution of amplitudes.

\begin{tabular}{|l|c|c|}
\hline \multicolumn{1}{|c|}{ Experimental Conditions } & Control/Active & Fraction Large Fluctuations \\
\hline$(\mathrm{dA})_{10}+\mathrm{dNTPs}+\mathrm{Mg}^{2+}$ & $\mathrm{A}$ & 0.69 \\
\hline$(\mathrm{dA})_{10}+\mathrm{dATP},+\mathrm{dCTP},+\mathrm{dGTP},-\mathrm{dTTP},+\mathrm{Mg}^{2+}$ & $\mathrm{C}$ & 0 \\
\hline$(\mathrm{dC})_{10}+\mathrm{dNTPs}+\mathrm{Mg}^{2+}$ & A & 0.52 \\
\hline$(\mathrm{dA})_{10}(\mathrm{dC})_{10}+\mathrm{dNTPs}+\mathrm{Mg}^{2+}$ & A & 0.44 \\
\hline$(\mathrm{dATC})_{5}+\mathrm{dNTPs}+\mathrm{Mg}^{2+}$ & A & 0.52 \\
\hline$(\mathrm{dA})_{10}(\mathrm{dC})_{10}-\mathrm{dNTPs}+\mathrm{Mg}^{2+}$ & $\mathrm{C}$ & 0 \\
\hline$(\mathrm{dA})_{10}(\mathrm{dC})_{10}+\mathrm{dNTPs}-\mathrm{Mg}^{2+}$ & $\mathrm{C}$ & 0 \\
\hline$(\mathrm{dA})_{10}(\mathrm{dC})_{10}+\mathrm{dTTP}-\mathrm{dGTP}^{2+}+\mathrm{Mg}^{2+}$ & $\mathrm{C}$ & 0.32 \\
\hline$(\mathrm{dA})_{10}(\mathrm{dC})_{10} \mathrm{NH}-\mathrm{dNTPs}+\mathrm{Mg}^{2+}$ & $\mathrm{C}$ & 0 \\
\hline No template $+\mathrm{dNTPs}+\mathrm{Mg}^{2+}$ & $\mathrm{C}$ & 0 \\
\hline
\end{tabular}

